Fecha de recepción: julio 2014

Fecha de aceptación: marzo 2015

Versión final: julio 2015

\section{Tendencias de consumo, innovación e identidad en la moda. Transformaciones en la enseñanza del diseño latinoamericano}

Verónica Fiorini *

\begin{abstract}
Resumen: Este texto se desarrolla en torno a la tensión entre los conceptos de tendencia e innovación en la construcción de las marcas identitarias de indumentaria en la Argentina. Se contemplarán estas problemáticas desde una perspectiva didáctica, proyectual y comunicacional dentro del campo del consumo y del diseño. La búsqueda de ciertos ejes discursivos claves para el desarrollo de los proyectos de indumentaria apuntarán a reformular ciertos preconceptos en torno a la idea de producto, consumo e identidad en la moda. La idea naturalizada de que las tendencias son "diseñadas" en las grandes capitales de la moda será puesta en discusión, para permitir habilitar otras estrategias de enseñanza del diseño, lejos de la copia, capaces de captar la creciente complejidad semántica dentro de un contexto globalizado y cambiante.
\end{abstract}

Palabras clave: diseño - tendencias - discursos - identidad - innovación - global - local.

[Resúmenes en inglés y portugués en la página 89]

${ }^{(*)}$ Diseñadora de Indumentaria (UBA). Especialista en Gestión de Diseño (UBA) y en Teoría del Diseño Comunicacional (UBA). Profesora Titular en Diseño VI en la Universidad de Palermo y Profesora Titular Regular en Diseño de Indumentaria y Textil, Diseño 1 y 2 en FADU-UBA. Coordinadora de la Carrera de Diseño de Indumentaria en UBA (2001-2006).

\title{
Introducción
}

Existen ciertos conceptos en el campo de la moda, que se presentan naturalizados dentro de la disciplina del diseño de indumentaria pero que indudablemente encierran algunos interrogantes dignos de ser revisados. Los fundamentos e ideas centrales que sustentan los proyectos de moda y aún los proyectos de marcas conviven con muchas de estas creencias que pocas veces son repensadas en el marco de un debate profundo en torno al diseño latinoamericano.

Términos como tendencias, innovación y modos de consumo, conforman una tríada que intentaremos explorar en relación al campo del proyecto y a como son pensados, tanto desde la óptica del mercado, como desde la formación académica de los diseñadores.

La idea de tendencia en la mirada de la prensa, de las marcas y de los estudiantes presenta algunas aristas capaces de ser discutidas y revisadas. En la primer parte del artículo, nos 
centraremos en los discursos que dan cuenta de ciertas ideas relacionadas con las tendencias en el campo del Sistema de la Moda. En la segunda parte, revisaremos esas ideas en términos de identidades vestimentarias. El término de identidades sociales es entendido desde la óptica del sociólogo Fred Davis (1994, p. 21) como fenómeno individual pero con un componente colectivo que las define. Este autor resalta la importancia de la ambivalencia de la identidad colectiva como una importante fuente cultural en los cambios vestimentarios que se producen en la moda. En los apartados posteriores, se profundizará en las relaciones entre moda y tendencias y brevemente en algunos de los alcances de estos cambios en la enseñanza de la disciplina del Diseño de Indumentaria.

\section{Moda, tendencias e innovación: algunos presupuestos disciplinares}

Desde la óptica de las marcas argentinas y desde el campo de la didáctica del diseño, el concepto de tendencia se argumenta con ciertos preconceptos o prejuicios que pueden sintetizarse en los siguientes ejes:

1. Las tendencias cuando son concebidas como fenómenos específicos del campo y que representan e irrumpen como nuevos e inesperados productos en términos de diseño y consumo. Los productos en el marco de las tendencias son presentados desde el periodismo de moda, las consultoras y aún desde la didáctica como ideas que plantean rupturas con el pasado, nuevas imágenes y estéticas a ser consumidas.

2. La idea de que las tendencias son decodificadas únicamente por los especialistas o consultores de tendencias, quiénes dan cuenta a las marcas de una suerte de glosario o dictado de aquellas formas, colores y texturas que serán las elegidas por la industria y el mercado en forma masiva. Desde otro punto de vista, se plantea en modo inverso, que las tendencias son aquellas que surgen de manera espontánea en la calle, a partir de estilos propuestos por los mismos usuarios, conformando modelos estéticos capaces de influenciar a los diseñadores, a las marcas y también a la crítica especializada que luego los difunde. Un ejemplo de este enfoque son aquellas tribus urbanas que devienen en imágenes y discursos representados en las marcas. Tal es el ejemplo del punk en el Reino Unido llevado al campo de la moda por Zandra Rhodes y Vivienne Westwood.

3. El preconcepto de que las tendencias provienen de los centros tradicionales del diseño (París, Milán, Londres, NY, Tokio). Pocas veces se sitúa la idea de tendencia como un fenómeno capaz de plantearse en otras latitudes como Sudamérica, y capaz de influenciar a otras capitales de la moda. Desde esta óptica, debemos reconocer a Alexander Hertcovitch y a Pablo Ramírez como dos ejemplos remarcables en su esfuerzo por situar al diseño latinoamericano en el mapa mundial de la moda.

4. Para Caldas (2004, p. 187); "En la segunda mitad del siglo XX, nuevas metrópolis se disputan el título de "capitales de la moda", entre las cuales figuran las ciudades latinoamericanas de San Pablo, Río de Janeiro, Bogotá y Buenos Aires". Este autor resalta que pese al creciente proceso de globalización, existe una emergencia y un rescate de las identidades locales en una nueva dinámica de influencias en un mercado global. En este proceso de internacionalización de la moda, Caldas remarca la importancia de preguntarnos qué 
buscar como referencia local dentro de un universo global. Existen numerosos ejemplos en donde se evidencia la idea de que Latinoamérica, recibe y resignifica las tendencias de otras latitudes. Pero aún es fuerte y profundo el mito de una cultura "desfasada" (una temporada atrás), un modelo en el cual numerosas marcas y consultoras locales de tendencias, sitúan la mirada en vidrieras, desfiles, sitios web, europeos, japoneses y norteamericanos. 5. Podemos remarcar un último preconcepto acerca de las tendencias, y es que son pensadas en su esencia como modas pasajeras y frívolas, que cambian la apariencia del producto sin mejorar su performance ni sus cualidades técnicas, formales y simbólicas. Desde esta óptica, lo que se propone no es una verdadera ruptura sino una cuestión de cambios "superficiales" o "cosméticos", reeditando cierta tensión histórica entre funcionalismo y styling.

Los cuatro aspectos que he mencionado, conforman una suerte de presupuestos disciplinares que argumentan de manera tácita los esquemas de enseñanza del diseño de indumentaria en la Argentina. El propósito es entonces desarticular ciertos conceptos del campo (Bourdieu) para poder visualizar algunas problemáticas vinculadas a los aspectos metodológicos en términos de construcción de los significados en los proyectos de diseño, excediendo las fronteras de lo estrictamente linguístico.

Algunos de estos subtemas serán problematizados en este artículo intentando aportar algunas reflexiones desde distintos puntos de vista. En primer lugar, se observa cierta tensión discursiva que se evidencia entre influencias globales y costumbres o improntas locales. En este sentido, la búsqueda de un diseño con identidad se cuestiona bajo una mirada que procesa influencias, pero que en muchos casos se cruza con el discurso acerca de la búsqueda de originalidad en el diseño argentino.

La búsqueda de diseños diferenciados, capaces de dar cuenta de un marco sociocultural local se enlaza con las prácticas de innovación en el diseño. Desde el punto de vista teórico trabajaremos y debatiremos para comprender la idea de tendencia con los enfoques de $\mathrm{F}$. Morace (2012) y D. Caldas (2004). Por otra parte, desde una mirada histórica, las relaciones entre términos como moda y tendencia así como el factor temporal en los procesos de diseño de indumentaria también merecen ser revisados a la luz de un mapeo de hábitos de consumo. Por último, se intentará delinear algunos aspectos sujetos a consideración hacia una búsqueda de estrategias de enseñanza que refuercen el concepto de innovación y diferenciación respecto de otros discursos de diseño (no sólo de otras morfologías o materialidades).

Considero que en el marco del diseño contemporáneo, asistimos a un cambio profundo en el campo de la indumentaria que propone un corrimiento en la búsqueda desenfrenada de nuevas versiones de los productos hacia una innovación más profunda, que excede el campo de las formas y se apoya en un sentido comunicacional. Esta búsqueda se evidencia en las nuevas concepciones acerca del fenómeno de las tendencias de consumo por parte de los diseñadores y consumidores.

$\mathrm{Al}$ referirnos al término "tendencia" adscribimos a la definición que F. Morace impuso en los años 90” por considerarla vigente:

En la fenomenología de la vida cotidiana se han evidenciado cada vez con más frecuencia patrones de comportamiento, entramados de valores y 
conjuntos de estilos de vida, que han adquirido paulatinamente una dignidad teórica y un nombre de pila: tendencias (trends) y megatendencias (megatrends). (1993)

En los últimos tiempos (2012) este autor ha reemplazado el término mega trends por el de escenarios de consumo caracterizando a los mismos como lapsos temporales de al menos diez años.

Consideramos interesante esta distinción, ya que desde la óptica de la enseñanza y desarrollo del diseño profesional, las megatendencias de consumo pueden aportar más elementos de análisis que las variables micro que proponen ciertas publicaciones especializadas en moda. También D. Caldas (2004, p. 217) propone definir el término tendencia como "una manifestación, en la esfera del comportamiento, del consumo o del 'espíritu del tiempo', de una sensibilidad anunciada por señales". Este autor brasilero remarca la importancia de la identificación de esta suerte de señales en el ámbito empresarial cuyo objetivo último es reducir el riesgo en el contexto del mercado.

Podemos afirmar que en la actualidad, algunos de los principales cambios en los hábitos de consumo están centrados en la búsqueda de discursos identitarios locales en diálogo simultáneo con el campo global de la moda.

En esta dirección, el sociólogo F. Morace (2012), identifica como una de las seis pautas del consumo emergente del último tiempo, el denominado "consumo arquetípico". Con este término se refiere a ciertas señales identitarias locales relacionadas con países, pueblos o naciones que aparecen fuertemente en los productos del consumo actual. También se refiere al consumo como "recuerdo vital", es decir como canal de expresión de culto a ciertas tradiciones de su lugar de origen. Pero en este punto, es necesario remarcar que desde nuestro punto de vista, no se trataría solamente de diseño con referencias locales sino de planteos proyectuales innovadores desde lo simbólico.

Estas transformaciones pueden evidenciarse en la Argentina en algunos casos emblemáticos del Buenos Aires Fashion Week edición invierno 2014, evento emblemático de la moda en nuestro país.

El caso de la diseñadora argentina Daniela Sartori propone en su colección "Bangladesh" un diálogo local con otras culturas en un sentido social. La misma propone recordar la tragedia ocurrida en los talleres de confección de la ciudad mencionada. Este cruce que expresa una tendencia dentro del multiculturalismo, suma una propuesta constructiva sumamente innovadora. Paños de tela de formas geométricas simples se transforman en reversiones del sari y otras tipologías hindúes. Desde el punto de vista conceptual, Sartori propone bajo el concepto de "copyleft" y las licencias Creative Commons, poner en crisis el concepto de autoría y del diseño como objeto de lujo, permitiendo a los usuarios reproducir sus diseños individualmente adquiriendo sus moldes e indicaciones técnicas a través de la computadora. Este ejemplo nos obliga a revisar el rol del diseñador, que en modo alguno se limita a un generador de formas o productos, sino que constituye verdaderamente un agente cultural innovador capaz de transformar ciertos hábitos de consumo y también modos de concebir los objetos. Esta diseñadora se opone a concebir objetos de diseño "de autor" como elementos exclusivos y en esta elección el discurso se enlaza con su propia cultura y sus condiciones de producción. Ella compara en forma explícita el trabajo de 
los talleres de la India con los de Buenos Aires y crítica el sistema de la moda en su lógica sociodeológica y en su contexto local.

Otro caso destacado es la alianza comercial y cultural entre Spazio Sumampa y Elementos Argentinos como Socios Solidarios presentes en BAFWEEK (2014).En esta propuesta, se difunde el trabajo de tejido del norte santiagueño a la vez que se propone cierta customización de alfombras a medida con ciertos diseños predeterminados en el contexto de una galería textil. En este ejemplo, existe un diálogo invaluable sobre la identidad argentina, y sobre un intercambio entre trabajadores textiles del interior del país y espacios relacionados al arte textil de Buenos Aires. Lo manual y lo artesanal se relacionan con lo artístico creando fenómenos y entrecruzamientos disciplinares entre artesanía-arte y diseño, y en donde lo importante, más allá de los objetos, es el mensaje conceptual de esa alianza.

En este sentido, se vuelve crucial el proceso de generación de los significados y de resignificación en los proyectos de diseño. En el primer ejemplo, en términos de indefinición de los límites entre diseñador y consumidor y de una posición crítica frente a los modos productivos y proyectuales tradicionales. En el segundo caso, la fusión entre lo artesanal y lo industrial (la resiginificación de lo autóctono en su convivencia con lo urbano).

\section{Algunas reflexiones sobre la identidad en el diseño}

Los fenómenos identitarios, por su carácter ambiguo y polisémico pueden presentarse en una primera instancia como inaprehensibles, opacos e indefinidos. Desde una óptica antropológica, Claude Levi-Strauss (1981), sostenía que el tema de la Identidad no se sitúa sólo en una encrucijada sino en varias. De modo inequívoco, afecta a todas las disciplinas, y también a todas las sociedades que estudian los etnólogos. En términos teóricos, podemos situar la problemática oscilando entre los aspectos particulares que distinguen a cada cultura y aquellos que están enmarcados en una mirada globalizadora y homogénea que desdibuja o anula las diferencias.

Etimológicamente el término identidad proviene del latín identitas, que en sentido filosófico, significa igualdad del ser respecto de sí mismo. Para Y. Zimmermann en la coherencia entre apariencia y ser, se manifiesta la identidad. Esto, sin embargo esconde un razonamiento válido, que es el que asocia la noción de identidad con la dimensión temporal: es posible afirmar que la identidad se construye en el tiempo. Desde esta óptica sería posible identificar ciertos rasgos formales y conceptuales invariantes a lo largo del tiempo, regidos por reglas o patrones que puedan comprobar la existencia de preferencias sociales en relación a determinadas formas, cortes, texturas, combinaciones, usos, etc. Siguiendo este razonamiento, se nos plantea un interrogante: ¿existe esta unidad entre nuestra manera de ser y nuestra forma de vestir? Esta mirada corre el riesgo de incurrir en un reduccionismo a ultranza, ya que la indumentaria proclama la mayoría de las veces no lo que somos sino lo que queremos ser, y eso también constituye una faceta esencial de la identidad porque implica una mirada que elige, que selecciona, aún cuando hablemos de influencias externas o de un contexto de globalización creciente.

Ciertas marcas argentinas como Cardón o La Dolfina, juegan con el imaginario del campo, de deportes como el polo y de ciertas tipologías de prendas vinculadas a una estética del 
gaucho tradicional, rasgos que son considerados típicos locales. Diseñadores del denominado diseño de autor como Pablo Ramírez han incursionado reiteradamente en temáticas nacionales como búsqueda original vinculada a la identidad local (espíritu colegial argentino vinculado a lo religioso y pueblerino).

Pero en cierto modo, desde una perspectiva didáctica es necesario ampliar la búsqueda en términos identitarios en un diálogo profundo con los hábitos de consumo y con las referencias internacionales. Se torna una mirada reduccionista aquella que limita la problemática de identidad a ciertos temas autóctonos, tipologías, materiales textiles o técnicas. Es imprescindible sumar los aspectos comunicacionales, conceptuales y estilísticos, y permitir nuevos enfoques o miradas originales sobre campos temáticos diversos. En esta concepción, se vislumbra una reflexión más amplia: establecer nuestras raíces en nuestros modos de pensar, de elegir, modos de procesar influencias, de rediseñar información del pasado y de otros contextos socioculturales. Para Jean Marie Benoist:

Una identidad grosera, inmediata, una identidad de superficie debe ceder el lugar a una investigación de las estructuras profundas que moldean la identidad en su aspecto relacional: la cuestión del "otro" aparece como constitutiva de la identidad. En este sentido, es importante establecer nuevamente la visión de conjunto, y la apreciación de rasgos diferenciales conceptuales que distingan los códigos propios de nuestra cultura. (Benoist, 1981)

En el caso de reconocidos diseñadores como Ronaldo Fraga, resulta evidente que la óptica brasilera no se traduce solamente en la elección de temas relativos a su nacionalidad, sino que es su enfoque del diseño y las connotaciones de sus diseños lo que evidencian un sentido del humor crítico y una estética de una sensualidad aniñada y artesanal que forma parte de cierta identidad latinoamericana. Son entonces su visión particular al construir significados y su concepción ideológica del diseño las que habilitan a pensar en términos de productos con ciertos rasgos de la identidad brasilera.

\section{Tiempo, modos de consumo e identidad: una narrativa en movimiento}

Para Z. Bauman (2007) en el pasaje de una sociedad de productores a una sociedad de consumidores, existe una transformación de los alcances temporales de los objetos de consumo. La perdurabilidad que se conformaba como anhelo dentro del primer modelo de sociedad moderna se vuelve un concepto que es preciso anular: la obsolescencia planificada se vuelve un imperativo y eso conlleva de por sí una mayor inestabilidad y aceleración de los deseos de consumo. Desde la óptica de este autor, entonces el tiempo ya no es cíclico ni lineal, sino que presenta rupturas y discontinuidades. En este contexto, $Z$. Bauman (2007, p. 74) destaca que el mercado lanza una oferta permanente a la manera de diversos kits de identidades para consumidores que son por otra parte ellos mismos objetos de consumo. Para este autor es el fetichismo de la subjetividad y no ya el fetichismo de la mercancía el que prevalece en la sociedad de consumidores. 
Esta idea central del autor nos permite preguntarnos, dentro de este esquema, acerca de las transformaciones que redefinen el rol de los diseñadores de moda. Bajo esta perspectiva, es posible pensar que el diseñador no construye un diseño o un producto sino un concepto identitario que se potencia en un ritmo de circulación cada vez más veloz. Frente a una cultura en donde prevalece el exceso de consumo a ritmos acelerados, los objetos de moda de corta duración empiezan a ser repensados desde el campo proyectual. Estas reflexiones no surgen solamente desde la creciente conciencia ambiental o de la búsqueda de diseños sutentables, sino también de modo contundente desde una búsqueda en el campo de la significación.

Para D. Crane (2000): "Las prendas son como textos abiertos que pueden adquirir todo el tiempo nuevos significados".

Propongo asociar esta nueva modalidad proyectual al concepto de una narrrativa en movimiento, lejos de un modelo estático, sino de un planteo conceptual capaz de dialogar con múltiples discursos (locales y globales) dentro y fuera de la disciplina. Esto también tiene su fundamento en los cambios en el campo del consumo. Los consumidores no esperan colecciones "cerradas" sino prendas de vestir capaces de intercambiar ideas con otros productos y otras marcas argentinas y de otras culturas. Ante todo, existe una búsqueda creciente de productos que conlleven una mirada innovadora sobre los productos, sus apropiaciones y su sentido comunicacional.

Un caso remarcable es el de la marca argentina Juana de Arco que propone en su identidad de diseño de autor, una búsqueda en la que funde aspectos artesanales, lúdicos y retro 70's, convirtiendo su narrrativa en un discurso capaz de ser consumido simultáneamente en Tokio y en Buenos Aires. Es decir, que hay un diálogo de comunión entre ciertas experiencias de consumidoras adolescentes en las comunidades de ambos países. El diseño, en este caso, es producido para distintos mercados, por ende con diferente significación en cada contexto de uso.

En el paradigma de la Modernidad, los productos tendían al mejoramiento en un marco de estandarización. En la Modernidad tardía se manifiesta una búsqueda contraria, una mayor diferenciación y personalización del consumo.

Esta transformación también contribuye a reforzar la idea de reflexión permanente sobre las marcas identitarias como uno de los ejes claves en los procesos de diseño. Del mismo modo, impacta en el acrecentamiento de los modelos de innovación (radical e incremental), debilitando la idea de aquellas propuestas de infinitas variaciones morfológicas desprovistas de sentido social y cultural.

En síntesis, considero que la identidad en términos vestimentarios debe ser pensada en forma dinámica, como una narrativa en movimiento construida en relación a otras voces discursivas que circulan simultáneamente y que no se presentan en modo alguno de manera nítida sino más bien yuxtapuesta.

\section{Diálogo Moda y tendencias: coincidencias y distinciones}

Pensar en términos de tendencias socioculturales de consumo, desde la visión de F. Morace implica un corrimiento de la escena específica de la moda para poder visualizar cam- 
bios a un nivel más amplio y en un franco diálogo con otras áreas. Lo cierto es que moda y tendencias tienen tres aspectos en común que son:

- Temporalidad: Una duración limitada ya que nunca se piensa en una moda o en una tendencia de consumo ilimitada en el tiempo. Por el contrario, los límites temporales se imponen como un imperativo.

- Cierto grado de opacidad: La aparente imprevisibilidad de estos fenómenos en el contexto industrial y sociocultural. Nuevos productos o hábitos de consumo parecen sorprender e irrumpir de modo sorpresivo en las prácticas de los consumidores, aún cuando esté institucionalizado el juego de la predicción de las tendencias de moda en esta práctica cultural.

- Diálogo mutuo: la moda influencia en su lógica otras prácticas culturales y otras esferas de la cultura configuran también sus lógicas y sus intereses.

Pero no todo lo que es considerado moda supone una tendencia. En general se supone que la tendencia es vanguardia anticipatoria y la moda establece un vínculo con lo masivo e industrial.

Para L. Svendsen (2006, p. 11): "La moda no es solo un asunto relativo al indumento, debe ser también considerada como un mecanismo o una ideología que es aplicable a casi todas las áreas del mundo moderno, desde la Edad Media en adelante". Para este autor en el marco de la aceleración del Sistema de la Moda, los cambios en los productos son "superficiales". Bajo esta óptica es difícil pensar en la innovación verdadera como fundamento del proyecto pero al mismo tiempo existe una renovación comunicacional altamente exigente (Fiorini, 2008). El mismo producto cambia de significado a veces en solo 8 semanas. Esa inestabilidad semántica forma parte de la lógica del Sistema de la Moda y también de las microtendencias en el campo del indumento. La pregunta ya no es entonces si se usará tal o cual producto en una temporada específica sino que significará en ese preciso momento para los consumidores.

La originalidad simbólica y la innovación formal se vuelven un escenario altamente complejo para el quehacer de los diseñadores de esta disciplina. En este contexto, marcas argentinas como HE (Hermanos Estebecorena) desafían este acelerado devenir de los productos cuando afirman que sus colecciones son continuaciones de sus productos anteriores. Esto plantea un desarrollo más reflexivo que apunta a una búsqueda de la innovación a largo plazo además de tener en cuenta una mayor conciencia ambiental.

Bajo este panorama, es lícito afirmar que los consumidores comienzan a exigir en las producciones de vestimenta de las marcas de diseño signos que hablen de la identidad en aquello que continúa siendo efímero por naturaleza.

Por eso es válido pensar que las tendencias actúan en el campo del diseño de Moda con la modalidad de tramas abiertas que dan entrada a los fenómenos de innovación e interpretación de signos detectados por los diseñadores en la generación misma de la colección de productos. Lejos de ser estáticas y de proponer la unicidad, se articulan con la mirada y la concepción y la narrativa del diseño que posea la marca, el diseñador o el estudiante de diseño. 


\section{La enseñanza del diseño: pensamiento e innovación}

El Oxford English Diccionary menciona por primera vez en 1588 el vocablo diseño al que define como un boceto o dibujo que sirve de modelo para su realización. Desde esta óptica el término aparece asociado a un plan mental, a una idea que es plasmada gráficamente. Considero apropiado distinguir, dentro del diseño de indumentaria dos instancias claves desde una óptica didáctica. (Fiorini, 2008, p. 96)

1. El diseño como proceso de pensamiento, reflexión e innovación

2. El objeto-vestimenta como resultado comunicacional, técnico y morfológico

A partir de esta doble categorización, podemos pensar los proyectos de diseño como procesos capaces de plantear proyectos que propongan recorridos comunicacionales móviles, capaces de mutar en lapsos cortos de tiempo su significado y potenciando su durabilidad conceptual en términos de innovación. Desde esta perspectiva, para los diseñadores ya no es imprescindible imaginar los escenarios de inserción de sus productos como contextos estáticos. Por lo tanto, en materia de didáctica del proyecto, la indumentaria nos coloca ante varios desafíos.

- Formar diseñadores críticos dentro del Sistema de la Moda, con proyectos capaces de adaptarse a contextos cambiantes, entendiendo al diseño inserto en el conjunto de las prácticas sociales. La indumentaria como código débil, no verbal, en el cual es difícil distinguir unidades concretas conforma un dispositivo que da un marco complejo de significación a la vestimenta. La articulación de este entramado simbólico de alta mutabilidad temporal obliga a formar a los diseñadores como verdaderos agentes comunicacionales más que como generadores formales de objetos vestimentarios. Estos diseños contemplarán diferente significación a nivel local y global y eso implica una mayor investigación que combina al campo del proyecto con la semiótica.

- La constante renovación de significados convierte a la disciplina del diseño de indumentaria constituye uno de los pilares más contundentes del campo, y este panorama se articula en la interrelación de diseñadores-usuarios-objetos-hábitos de consumo. Es precisamente éste recorrido el que el diseñador debe analizar y replantear, no en un sentido "predictivo" sino reflexivo y estratégico, anticipando variaciones del gusto y de las costumbres en términos de innovación y valor agregado. Las tendencias socioculturales de consumo suponen un constante relevo de los deseos de los consumidores a favor de ciertas concepciones, prácticas y objetos. En esta intersección se sitúa claramente uno de los nodos centrales de la práctica del diseño en el marco de la contemporaneidad.

- En este contexto de tendencias de consumo, se evidencia una búsqueda remarcable y acrecentada en las últimas décadas de ciertas marcas identitarias en los proyectos de diseño. Desde la óptica proyectual la identidad no aparece como objetivo último del diseño sino como parte del proceso. Es el enfoque de una narración dinámica en el diseño lo que supone, no sólo una búsqueda limitada de materiales o técnicas autóctonas, sino una búsqueda conceptual y simbólica, capaz de aunar lo propio en diálogo franco con 
otras latitudes. Este tipo de proceso de diseño es un rasgo constituyente de la identidad más profunda en términos culturales.

\section{A modo de recapitulación}

Hemos revisado algunas concepciones en torno a la problemática de tendencias de consumo y su impacto en el Sistema de la Moda. En este sentido, advertimos que era necesario pensar en las prácticas de diseño latinoamericano como búsquedas legítimas de innovación en términos de significación y definición de la identidad, y no como meras transformaciones formales. También hemos podido ampliar la mirada sobre lo que suponen los rasgos identitarios en la moda, focalizando en la posición ideológica de los diseñadores de una región, sin reducirlas exclusivamente a las búsquedas folklóricas tradicionales.

Por último, nos adentramos en el campo metodológico para pensar en los proyectos de diseño como narrativas en movimiento, capaces de dialogar con los cambios permanentes en los hábitos de consumo. De este modo abierto, el pensamiento de diseño se articula con la interpretación de tendencias y con la lógica de la moda.

En síntesis, es preciso ampliar el debate en el campo de la enseñanza del disenõ de indumentaria, en relación a un sistema basado en la obsolescencia planificada de los productos y en su estacionalidad. También es necesario pensar estrategias de soporte en un contexto de creciente complejidad semántica para mercados micro segmentados y altamente informados. Dadas las condiciones mencionadas anteriormente, es lícito pensar que la enseñanza de la metodología de diseño no puede seguir un esquema lineal sino que debe asumir una postura más compleja y articularse con saberes del orden de la comunicación y de la interpretación de ciertas señales (Caldas) que anticipan cambios culturales y que definen un contexto local.

Este artículo lejos de pretender esclarecer esta problemática, deja abierto un debate, cierta dialéctica en la cual no existen juicios de valor sino un entrecruzamiento complejo. Allí justamente confluyen los modos de procesar influencias, de resignificar lo propio y de rescatar la propia cultura.

\section{Referencias Bibliográficas}

Barthes, R. (2003). El sistema de la moda y otros escritos. Buenos Aires: Paidós.

Bauman, Z. (2007).Vida de consumo. Buenos Aires: Editorial Fondo de Cultura Económica. Benoist, Jean Marie (1981). La identidad. Madrid: Ediciones Petrel.

Burdek, B. E. (1994). Diseño. Historia, teoría y práctica del diseño industrial. Barcelona: Editorial GG Diseño,.

Caldas, D.(2004). Observatorio de Sinais. Teoría e prática da pesquisa de tendencias. Río de Janeiro: Editorial Senac Rio.

Davis, Fred (1992).Fashion, culture and identity. Editorial The University of Chicago Press: Chicago. 
Fiorini, V. et al (2008). Design de moda.Olhares diversos. En Pires, D. (Comp). Design de Moda:abordagens conceituais e metodológicas (p. 96). San Pablo: Editorial Estação das Letras e Cores.

Levi-Strauss, C. (1981). La identidad. Madrid: Editorial Petrel.

Morace, F. (2012). Una mirada al futuro. Entrevista a Francesco Morace. Diseño Producto. Revista digital d[x] i magazine n. 21, sección entrevistas. Disponible en: http://www.dximagazine. $\mathrm{com} / \mathrm{seccion} /$ entrevistas/interview-diseno/francesco-morace.--una-mirada-al-futuro

Morace, F. (1993). Contratendencias. Una nueva cultura del consumo. Madrid: Editorial Celeste Ediciones.

Svendsen, L. (2006). Fashion. A philosophy. Londres: Editorial Reaktion Books Ltd.

Summary: The text is developed around the tension between the concepts trend and innovation in the construction of fashion identity brands in Argentina. In the article, we contemplate these issues from a didactic, projective and communicational perspective within the fields of consumption and design. The searching for certain key design discursive axes for the fashion projects development will aim to reformulate certain preconceptions about the product idea and identity in fashion. The widespread idea of trends "being designed" in big fashion capitals will be questioned to consider other design education strategies, far from copying, able to capture the increasing semantic complexity in a globalized and changing context.

Key words: design - trends - speeches - identity - innovation - global - local.

Resumo: Este texto desenvolve-se em torno à tensão entre os conceitos de tendência e inovação na construção das marcas identitárias de indumentária na Argentina. Contemplar-se-ão estas problemáticas desde uma perspectiva didática, projetual e comunicacional no campo do consumo e do design. A procura de eixos discursivos chaves para o desenvolvimento dos projetos de indumentária apontarão a reformular preconceitos em torno à idéia de produto, consumo e identidade na moda. A idéia naturalizada de que as tendências são desenhadas nas grandes capitais da moda será posta em discussão, para permitir habilitar outras estratégias de ensino do design, longe da cópia, capazes de captar a crescente complexidade semântica dentro de um contexto globalizado e mutável.

Palavras chave: design - tendências - discursos - identidade - inovação - global - local. 